\title{
A 4-node plane element based quadrilateral area coordinate method QACM-II
}

\author{
Xiaoming Chen ${ }^{a}$, Jin Duan, Yungui Li \\ China state construction technical center, Beijing, China \\ aHanee@126.com
}

\begin{abstract}
Keywords: Finite element, Quadrilateral area coordinate, Generalized conforming
Abstract. Nonlinear transformation between the isoparametric and the Cartesian coordinates may make the elements more sensitive to mesh distortion. By using the second kind of quadrilateral coordinate method QACM-II, which can keep the transformation always linear between the area and the Cartesian coordinate systems, a 4-node plane element ACQ $\lambda 2$ was developed based on optimized generalized conforming conditions. Additional displacement field based on internal parameters was also used to make the whole displacement field quadratic complementary.
\end{abstract}

\section{Introduction}

As a local coordinate system, isoprametric coordinate method was widely used for developing finite elements as its convenience, such as the most famous elements Q6[1] and QM6[2], but the nonlinear transformation between the isoparametric and the Cartesian coordinates would lead to a problem that the serendipity family was quite sensitive to the mesh distortions.

For overcoming this inherent defect of isoparametric coordinates, Long et al. [3, 4] developed the first kind of quadrilateral area coordinate method QACM-I. For this kind of quadrilateral coordinate method, the transformation between the area and the Cartesian coordinate systems is always linear, so it is powerful for developing new elements which are much more insensitive to mesh distortion.

By using QACM-I, Chen et al. [5] proposed 4-node quadrilateral membrane elements AGQ6-I and AGQ6-II, which are locking free of MacNeal's thin beam. Based on this research, Cen et al. [6] derived out the analytical element stiffness matrix of AGQ6-I and developed a family of the quadrilateral plane membrane elements [7].

As QACM-I contains four area coordinate components $\left(L_{1}, L_{2}, L_{3}, L_{4}\right)$, among which only two are independent, Chen et al. [8] proposed the second kind of quadrilateral coordinate method QACM-II which has only two independent coordinate components $Z_{1}$ and $Z_{2}$.

In this paper, based on generalized conforming theory[9], a 4-node plane element ACQ 2 was developed by using QACM-II. Its nodal displacement field was formulated with optimized conforming conditions which would introduce fewest restrains on element's sides, and additional displacement field based on internal parameters was used to make the whole displacement field quadratic complementary.

\section{Generalized conforming conditions}

According to the theory of generalized conforming, the conforming conditions for plane elements under constant stresses are as follows:

Compatibility of constant stress $\sigma_{x}$ :

Compatibility of constant stress $\sigma_{y}$ :

$$
\iint \varepsilon_{x} d A \equiv \iint \frac{\partial u}{\partial x} d A \equiv \oint l u d s=\oint l u \tilde{d} d s
$$

$$
\iint \varepsilon_{y} d A \equiv \iint \frac{\partial v}{\partial v} d A \equiv \oint m v d s=\oint m \tilde{v} d s
$$

Compatibility of constant stress $\tau_{x y}$ : 


$$
\iint \gamma_{x y} d A \equiv \iint\left(\frac{\partial u}{\partial y}+\frac{\partial v}{\partial x}\right) d A \equiv \oint(m u+l v) d s=\oint(m \tilde{u}+l \tilde{v}) d s
$$

Compatibility of rotation:

$$
\iint 2 \varpi d A \equiv \iint\left(\frac{\partial v}{\partial x}-\frac{\partial u}{\partial y}\right) d A \equiv \oint(l v-m u) d s=\oint(l \tilde{v}-m \tilde{u}) d s
$$

Compatibility of motion along $x$ :

$$
\sum_{i=1}^{4}(u-2)_{i}=0
$$

Compatibility of motion along $y$ :

$$
\sum_{i=1}^{4}\left(v-\vartheta \phi_{i}=0\right.
$$

Eq.3 and Eq. 4 can be written in equivalent form as follows:

$$
\begin{aligned}
& \iint \frac{\partial u}{\partial y} d A \equiv \oint m u d s=\oint m \tilde{u} d s \\
& \iint \frac{\partial v}{\partial x} d A \equiv \oint l v d s=\oint l v \tilde{v} d s
\end{aligned}
$$

Then there are three conforming conditions for $u$ and $v$ respectively, and for a 4-node element, the additional condition was selected as follows:

$$
\begin{aligned}
& \sum_{i=1}^{4}(u-\mathcal{Q})_{i} \xi_{i} \eta_{i}=0 \\
& \sum_{i=1}^{4}(v-\vartheta)_{i} \xi_{i} \eta_{i}=0
\end{aligned}
$$

\section{Formulation of nodal displacement}

The nodal displacement field of the new element can be assumed with QACM-II as follows:

$$
\begin{aligned}
& u_{q}=\alpha_{1}+\alpha_{2} Z_{1}+\alpha_{3} Z_{2}+\alpha_{4} Z_{1} Z_{2} \\
& v_{q}=\beta_{1}+\beta_{2} Z_{1}+\beta_{3} Z_{2}+\beta_{4} Z_{1} Z_{2}
\end{aligned}
$$

With the conforming conditions presented in Eq.1 Eq.8, the undetermined parameters can be formulated out.

Substitute the results of $\alpha_{i}(i=1,2,3,4)$ into Eq.9, then the shape functions of nodal displacement can be written as:

$$
\begin{aligned}
N_{q i}= & \frac{1+3\left(g_{1} g_{3}-g_{2} g_{4}\right) g_{j} \xi_{i} \eta_{i}}{4\left(1+g_{1} g_{3}+g_{2} g_{4}\right)}+\frac{\xi_{i}}{4}\left[1-\frac{\eta_{i}\left(g_{3}-g_{2}\right) g_{j}}{1+g_{1} g_{3}+g_{2} g_{4}}\right] Z_{1} \\
& +\frac{\eta_{i}}{4}\left[1-\frac{\xi_{i}\left(g_{2}-g_{1}\right) g_{j}}{1+g_{1} g_{3}+g_{2} g_{4}}\right] Z_{2}+\frac{3 \xi_{i} \eta_{i} g_{j}}{4\left(1+g_{1} g_{3}+g_{2} g_{4}\right)} Z_{1} Z_{2}
\end{aligned}
$$

The Partial derivatives of shape functions are as follows: 


$$
\begin{aligned}
\frac{\partial N_{q i}}{\partial x} & =\frac{\xi_{i} \bar{b}_{1}}{4 A}\left[1-\frac{\eta_{i}\left(g_{3}-g_{2}\right) g_{j}}{1+g_{1} g_{3}+g_{2} g_{4}}\right]+\frac{\eta_{i} \bar{b}_{2}}{4 A}\left[1-\frac{\xi_{i}\left(g_{2}-g_{1}\right) g_{j}}{1+g_{1} g_{3}+g_{2} g_{4}}\right] \\
& +\frac{3 \xi_{i} \eta_{i} g_{j}}{4\left(1+g_{1} g_{3}+g_{2} g_{4}\right)}\left(\frac{\overline{b_{1}}}{A} Z_{2}+\frac{\overline{b_{2}}}{A} Z_{1}\right) \\
\frac{\partial N_{q i}}{\partial y} & =\frac{\xi_{i} \bar{c}_{1}}{4 A}\left[1-\frac{\eta_{i}\left(g_{3}-g_{2}\right) g_{j}}{1+g_{1} g_{3}+g_{2} g_{4}}\right]+\frac{\eta_{i} \bar{c}_{2}}{4 A}\left[1-\frac{\xi_{i}\left(g_{2}-g_{1}\right) g_{j}}{1+g_{1} g_{3}+g_{2} g_{4}}\right]\left(\begin{array}{ll}
i=1,3,4 & j=i+2 \\
\text { when } i=3, & j=1 \\
\text { when } i=4, j=2
\end{array}\right) \\
& +\frac{3 \xi_{i} \eta_{i} g_{j}}{4\left(1+g_{1} g_{3}+g_{2} g_{4}\right)}\left(\frac{\overline{c_{1}}}{A} Z_{2}+\frac{\overline{c_{2}}}{A} Z_{1}\right)
\end{aligned}
$$

Then the matrix of strains $\boldsymbol{B}_{q}$ can be formulated out. The stiffness matrix of nodal displacement field can be written as:

$$
\boldsymbol{K}_{q}=\iint_{A} \boldsymbol{B}_{q}^{T} \boldsymbol{D} \boldsymbol{B}_{q} t d A
$$

Where $\boldsymbol{D}$ is the elastic matrix and $t$ is the thickness of element.

\section{Formulation of additional displacement}

Additional displacement field is assumed as follows:

$$
\begin{aligned}
& u_{\lambda}=\lambda_{1} N_{\lambda 1}+\lambda_{2} N_{\lambda 2} \\
& v_{\lambda}=\lambda_{1}^{\prime} N_{\lambda_{1}}+\lambda_{2}^{\prime} N_{\lambda 2}
\end{aligned}
$$

The shape functions of internal parameters can be selected as follows:

$$
\begin{aligned}
& N_{\lambda 1}=Z_{1}^{2}-\frac{2}{3}\left(g_{2}-g_{1}\right) Z_{1}-\frac{1}{3}\left[\left(g_{3}-g_{2}\right)^{2}+1\right]+\frac{2}{9}\left(g_{2}-g_{1}\right)^{2} \\
& N_{\lambda 2}=Z_{2}^{2}-\frac{2}{3}\left(g_{3}-g_{2}\right) Z_{2}-\frac{1}{3}\left[\left(g_{2}-g_{1}\right)^{2}+1\right]+\frac{2}{9}\left(g_{3}-g_{2}\right)^{2}
\end{aligned}
$$

These two shape functions both can satisfy the conforming condition as:

$$
\begin{aligned}
& \iint \frac{\partial N_{2 i}}{\partial x} d A=\oint l N_{2 i} d s=0 \\
& \iint \frac{\partial N_{2 i}}{\partial y} d A=\oint m N_{2 i} d s=0 \\
& \iint N_{2 i} d A=0
\end{aligned}
$$

With static condensation method, the stiffness matrix of element can be written as:

$$
\begin{aligned}
\boldsymbol{K}^{e} & =\boldsymbol{K}_{q}-\boldsymbol{K}_{\lambda q}^{T} \boldsymbol{K}_{\lambda \lambda}^{-1} \boldsymbol{K}_{\lambda q} \\
\boldsymbol{K}_{\lambda \lambda} & =\iint_{A} \boldsymbol{B}_{\lambda}{ }^{T} \boldsymbol{D} \boldsymbol{B}_{\lambda} t d A \\
\boldsymbol{K}_{\lambda q} & =\iint_{A} \boldsymbol{B}_{\lambda}{ }^{T} \boldsymbol{D} \boldsymbol{B}_{q} t d A
\end{aligned}
$$

where $\boldsymbol{B}_{\lambda}$ is the strain matrix about $N_{\lambda i}$, it can be formulated with partial derivatives as follows:

$$
\begin{array}{ll}
\frac{\partial N_{\lambda 1}}{\partial x}=\frac{2 \bar{b}_{1}}{A} Z_{1}-\frac{2 \bar{b}_{1}}{3 A}\left(g_{2}-g_{1}\right) & \frac{\partial N_{\lambda 2}}{\partial x}=\frac{2 \bar{b}_{2}}{A} Z_{2}-\frac{2 \bar{b}_{2}}{3 A}\left(g_{3}-g_{2}\right) \\
\frac{\partial N_{\lambda 1}}{\partial y}=\frac{2 \bar{c}_{1}}{A} Z_{1}-\frac{2 \bar{c}_{1}}{3 A}\left(g_{2}-g_{1}\right) & \frac{\partial N_{\lambda 2}}{\partial y}=\frac{2 \bar{c}_{2}}{A} Z_{2}-\frac{2 \bar{c}_{2}}{3 A}\left(g_{3}-g_{2}\right)
\end{array}
$$

This element model is denoted as ACQ $\lambda 2$. 


\section{Numerical examples}

\section{Example 1. Patch test}

The constant strain/stress patch test is shown in Fig.1. Let Young' s modulus E=1000, Poisson's ratio $\mu=0.25$, and thickness of the patch $t=1$. ACQ $\lambda 2$ can present exact solutions as listed in Tab.1.

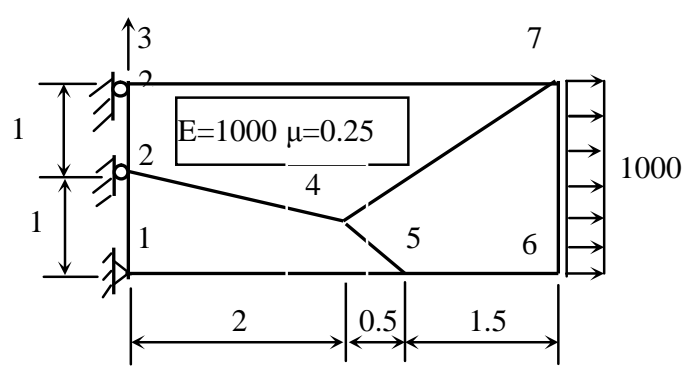

Fig.2 Patch test

\begin{tabular}{ccc}
\multicolumn{3}{c}{ Tab.1 Exact solutions of Patch test } \\
\hline Node & $u_{i}$ & $v_{i}$ \\
\hline 1 & 0.0 & 0.0 \\
2 & 0.0 & -0.25 \\
3 & 0.0 & -0.5 \\
4 & 2.0 & -0.125 \\
5 & 2.5 & 0.0 \\
6 & 4.0 & 0.0 \\
7 & 4.0 & -0.5 \\
\hline
\end{tabular}

Example 2. Cook's skew beam

A skew cantilever beam under shear distributed load at the free edge, as shown in Fig. 2, was proposed by Cook et al. [10]. The results of vertical deflection at point $\mathrm{C}$, the maximum principal stress at point $\mathrm{A}$ and the minimum principal stress at point $\mathrm{B}$ are listed in Table 2.

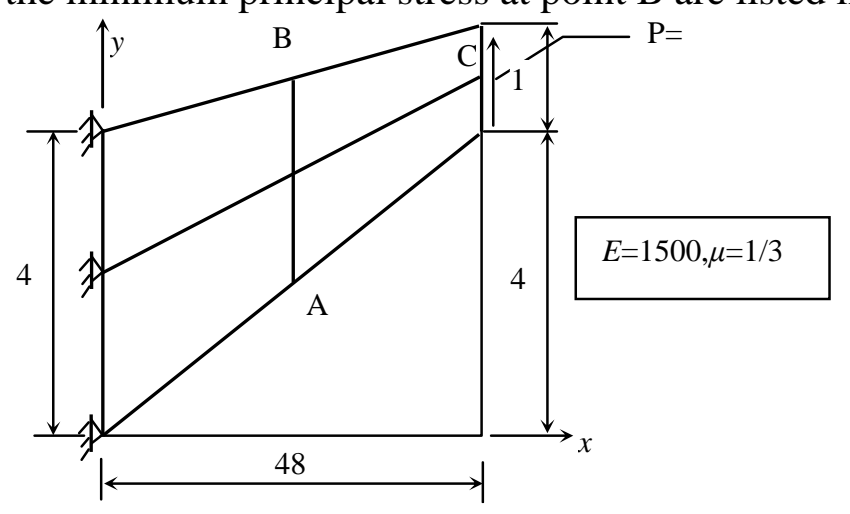

Fig.2 Cook's skew beam

Tab.2 Results of Cook's skew beam

\begin{tabular}{|c|c|c|c|c|c|c|c|c|c|}
\hline \multirow[b]{2}{*}{ Element } & \multicolumn{3}{|c|}{$\mathrm{V}_{\mathrm{C}}$} & \multicolumn{3}{|c|}{$\sigma_{\text {Amax }}$} & \multicolumn{3}{|c|}{$\sigma_{\mathrm{Bmin}}$} \\
\hline & $2 \times 2$ & $4 \times 4$ & $8 \times 8$ & $2 \times 2$ & $4 \times 4$ & $8 \times 8$ & $2 \times 2$ & $4 \times 4$ & $8 \times 8$ \\
\hline $\mathrm{Q} 4$ & 11.80 & 18.29 & 22.08 & 0.1217 & 0.1873 & 0.2242 & -0.0960 & -0.1524 & -0.1869 \\
\hline QM6 & 21.05 & 23.02 & - & 0.1928 & 0.2243 & - & -0.1580 & -0.1856 & - \\
\hline $\mathrm{HL}^{[11]}$ & 18.17 & 22.03 & 23.39 & 0.1582 & 0.1980 & 0.2205 & -0.1335 & -0.1770 & -0.1931 \\
\hline$P-S^{[12]}$ & 21.13 & 23.02 & 23.88 & 0.1854 & 0.2241 & 0.2364 & - & - & - \\
\hline ACQ $\lambda 2$ & 20.74 & 22.99 & 23.69 & 0.1936 & 0.2256 & 0.2345 & -0.1452 & -0.1866 & -0.1987 \\
\hline
\end{tabular}

Example 3. Cantilever beam divided by five quadrilateral elements

A cantilever beam divided by five irregular quadrilateral elements is shown in Fig.3. Two loading cases are considered: (a) pure bending under moment $M=2000$; (b) shear bending under transverse force $P=150$. The Young's modulus $E=1500$ and Poisson's ratio $\mu=0.25$. The results of the vertical defection $v_{\mathrm{A}}$ at point $\mathrm{A}$ and the stress $\sigma_{\mathrm{xB}}$ at point $\mathrm{B}$ are given in Tab. 3 . 


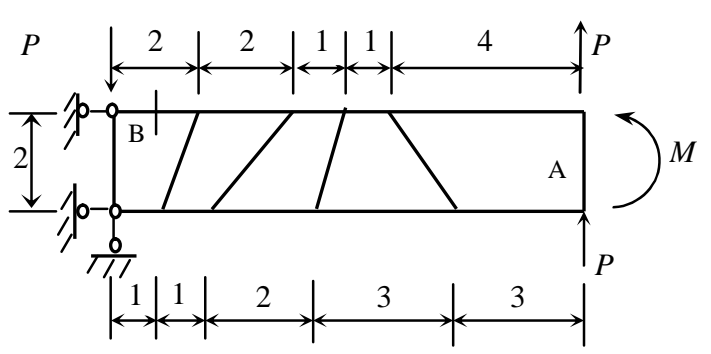

Fig.3 Cantilever beam with five irregular olamants
Tab.3 Results of cantilever beam

\begin{tabular}{|c|c|c|c|c|}
\hline \multirow[t]{2}{*}{ Element } & \multicolumn{2}{|c|}{$M$} & \multicolumn{2}{|c|}{$P$} \\
\hline & $v_{\mathrm{A}}$ & $\sigma_{\mathrm{xB}}$ & $v_{\mathrm{A}}$ & $\sigma_{\mathrm{xB}}$ \\
\hline $\mathrm{Q} 4$ & 45.7 & -1761 & 50.7 & -2448 \\
\hline QM6 & 96.1 & -2497 & 98.0 & -3235 \\
\hline $\mathrm{QC6}^{[13]}$ & 96.1 & -2439 & 98.1 & -3339 \\
\hline $\mathrm{NQ6}^{[14]}$ & 96.1 & -2439 & 98.0 & -3294 \\
\hline P-S & 96.2 & -3001 & 98.1 & -3899 \\
\hline $\mathrm{ACQ} \lambda 2$ & 96.0 & -3015 & 98.0 & -4135 \\
\hline Exact & 100 & -3000 & 102.6 & -4050 \\
\hline
\end{tabular}

\section{Conclusions}

By using the second kind of quadrilateral coordinate method QACM-II, a 4-node plane element ACQ $\lambda 2$ was developed based on generalized conforming theory. Optimized conforming conditions were selected to make the nodal displacement field keep lowest order and fewest restrains were applied on element's sides. Additional displacement field based on internal parameters was also used to make the whole displacement field quadratic complementary.

\section{References}

[1] E L Wilson, R L Taylor, et al., Incompatible displacement models, Numerical and Computer Methods in Structural Mechanics, In: S J Fenves et al., eds, Academic Press, New York, 1973, 43-57. [2] R. L. Taylor, P. J. Beresford, E. L. Wilson. A non-conforming element for stress analysis. International Journal for Numerical Methods in Engineering, Vol. 10, pp. 1211-1219, 1976.

[3] Y.Q. Long, J.X. Li, Z.F. Long, and S. Cen. Area coordinates used in quadrilateral elements. Communications in Numerical Methods in Engineering, vol. 15, no. 8, pp. 533-545, 1999.

[4] Z.F. Long, J.X. Li, S. Cen, and Y.Q. Long. Some basic formulae for Area coordinates used in quadrilateral elements. Communications in Numerical Methods in Engineering, vol. 15, no. 12, pp. 841-852, 1999.

[5] X.-M. Chen, S. Cen, Y.-Q. Long and Z.-H. Yao, "Membrane elements insensitive to distortion using the quadrilateral area coordinate method", Computers \& Structures, vol. 82, no. 1, pp. 35-54, 2004.

[6] S. Cen, Y Du, X.-M. Chen and X.-R. Fu, "The analytical element stiffness matrix of a recent 4-node membrane element formulated by the quadrilateral area coordinate method", Communications in Numerical Methods in Engineering, vol. 23, no. 12, pp. 1095-1110, 2007.

[7] S. Cen, X.-M. Chen and X.-R. Fu, "Quadrilateral membrane element family formulated by the quadrilateral area coordinate method", Computer Methods in Applied Mechanics and Engineering, vol. 196, no. 41-44, pp. 4337-4353, 2007.

[8] X.M. Chen, S. Cen, X.R. Fu, and Y.Q. Long, "A new quadrilateral area coordinate method (QACM-II) for developing quadrilateral finite element models," International Journal for Numerical Methods in Engineering, vol. 73, no. 13, pp. 1911-1941, 2008.

[9] Y.Q. Long, M.F. Huang. A generalized conforming isoparametric element. Appl. Math. Mech. -English Edition 9(10), pp. 929-939, 1988.

[10] Cook RD, Malkus DS, Plesha ME. Concepts and applications of finite element analysis. 3rd ed. New York: John Wiley \& Sons Inc.; 1989.

[11] Cook RD. Improved two-dimension finite element. J Struct Div, Asce 1974; 100ST9:1851-63.

[12] T.H.H. Pian and K.Sumihara, Rational approach for assumed stress finite elements. International Journal for Numerical Methods in Engineering, vol. 20, no. 9, pp. 1685-1695, 1984.

[13] W. J. Chen, L. M. Tang. Isoparametric quasi-conforming element. Journal of Dalian University of Technology, Vol. 20, no. 1, pp. 63-74, 1981.

[14] T.H.H. Pian, C. C. Wu. Hybrid and Incompatible Finite Element Methods. Chapman \& Hall/CRC, Boca Raton, 2006. 